Research Article

\title{
Knowledge of family planning and current use of contraceptive methods among currently married women in Uttar Pradesh, India
}

\author{
Saraswati Kerketta ${ }^{1}$, Ajay Kumar ${ }^{2} *$
}

\author{
${ }^{1}$ Department of Geography, Nistarini College, Puruliya, West Bengal, India \\ ${ }^{2}$ Town and Country Planning Organisation, E Block, Vikas Bhawan, I P Estate, New Delhi, India
}

Received: 29 September 2015

Accepted: 05 October 2015

\author{
*Correspondence: \\ Ajay Kumar, \\ E-mail: jnu.ajay@gmail.com
}

Copyright: (c) the author(s), publisher and licensee Medip Academy. This is an open-access article distributed under the terms of the Creative Commons Attribution Non-Commercial License, which permits unrestricted non-commercial use, distribution, and reproduction in any medium, provided the original work is properly cited.

\begin{abstract}
Background: India was the first country to implement population policies, still after 63 years, it's far away from achieving low fertility rates. Fertility rates are directly associated with the use of family planning methods. Besides, the use of contraceptive methods amongst the currently married women is very low (49\%) in India.

Methods: Data have been extracted from NFHS 2005-06 for Uttar Pradesh. Cross-tabulation and multivariate analysis have been carried out to study the patterns of contraceptive methods and their determinants.

Results: In Uttar Pradesh, CPR is very low (43\%). Women from rural area are $32 \%$ (OR=0.681**) less likely to use contraception than urban women. Women's age is positively related with current use of contraceptives. The likelihood of using contraception is $46 \%(\mathrm{OR}=0.549)$ lower among Muslims compared to Hindus. Women below middle school are $30 \%(\mathrm{OR}=1.302 * * *)$ and women with middle complete are $67 \%(\mathrm{OR}=1.669 * * *)$ more likely to use the contraceptive methods than the illiterate women. Women belonging to low SLI are $44 \%(\mathrm{OR}=0.569 *)$ less likely and with high SLI are $60 \%(\mathrm{OR}=1.5999 *)$ more likely to use contraception, than women belong to medium SLI. Women use more contraception with increasing parity. Among all the predictors women's education found to be the most important.

Conclusions: There is a wide gap between knowledge and acceptance of contraceptives among currently married women in Uttar Pradesh. There is an urgent need to strengthen the awareness among currently married women so that ultimate aim of reducing fertility rates can be achieved.
\end{abstract}

Keywords: Fertility, Contraceptive prevalence rate, Socio-economic predictors, Uttar Pradesh

\section{INTRODUCTION}

The latest report of United Nations (2015)-“World Population Prospects: The 2015 Revision" discloses that the current population of the world $(7.3$ billion $)$ is expected to reach 8.5 billion by $2030,9.7$ billion in 2050 and 11.2 billion in $2100 .{ }^{1}$ Most of the projected increase in the world's population can be attributed to a short list of countries having high-fertility rates. During 20152050 , half of the world's population growth is estimated to be concentrated in nine countries: India, Nigeria, Pakistan, Democratic Republic of the Congo, Ethiopia,
United Republic of Tanzania, United States of America (USA), Indonesia and Uganda. China and India will remain the two largest countries in the world, both with more than 1 billion population and representing 19 and 18 per cent of the world's population, respectively. But by 2022 , the population of India is expected to surpass that of China. According to the new projection, India will overtake China's population six years earlier than the previously predicted.

India was the first country in the world to adopt population policies in 1951 and launch National Family 
Planning Programme in the following year. After gaining independence the government of India realized that the rapid population growth in India was to be too high for realization of social and economic transformation of its people. The family planning programme in India has advanced through number of stages and has changed strategies to control the rapid population growth. Though fertility rates have come down but still Total Fertility Rate (TFR) is above replacement level. ${ }^{2}$ Fertility rates are directly associated with the use of family planning methods. The use of contraceptive methods is an important determinant which affects fertility levels in a region. Fertility rates as well as contraceptive prevalence rates vary significantly from one region to another and from one state to another. The north-south contraceptive divide is reflected in their TFR. In India, the knowledge of contraceptive methods, as evident from NFHS-III, is universal but there exists a huge gap when it comes to practice. $^{3}$ Despite of widespread knowledge of family planning methods, the use of modern methods is as low as 49 per cent amongst the currently married women. ${ }^{4}$ It reflects that around $51 \%$ of currently married women are not using modern contraceptive methods in India.

Empowered Action Group (EAG) states account for half of the country's population. Population giant of India, Uttar Pradesh is currently home of 16 per cent of India's total population. ${ }^{5}$ Every year the state adds more people to its population than any other state in India. Uttar Pradesh has been in the centre of various population stabilization programmes in India. In the last 65 years, Uttar Pradesh has experienced rapid population growth. ${ }^{6}$ Decadal population growth rate touched its peak $(25.8$ per cent) during 1991-2001; thereafter the decadal growth rate witnessed a decline by five points in the following decade. Total Fertility Rate for Uttar Pradesh was reported as 3.5 (NHHS-III) which was about 32\% higher than the national average of 2.7 .

In Uttar Pradesh the knowledge of contraception is almost universal; with $98 \%$ of currently married women having knowledge of at least one contraceptive method. ${ }^{7}$ The history of family planning programme indicates its focus on permanent methods of contraception. The huge gap between knowledge and acceptance of contraception, especially modern methods of contraception is salient. Female sterilization is the most widely known method of contraception in Uttar Pradesh with $98.9 \%$ and followed by male sterilization which is known to be $91.2 \%$ by currently married women. Knowledge of spacing methods is less than permanent methods. The contraceptive prevalence rate (CPR) is also very low, about $43.6 \%$, and contraceptive prevalence rate of modern method is as low as $29.3 \%$. $^{7}$

This paper explores the knowledge of different contraceptive methods among currently married women in Uttar Pradesh. An attempt has been made to examine the pattern of contraception use in Uttar Pradesh. Further an analysis of the socio-economic differentials and the factors affecting the use of contraceptive methods in Uttar Pradesh has been carried out.

\section{METHODS}

Data for the study has been taken from the National Family Health Survey (2005-06) for Uttar Pradesh. In Uttar Pradesh, NFHS-III, information was collected from 10026 households and 12183 eligible women were interviewed. NFHS-III used three type questionnaires: the household questionnaires, the women questionnaires, and the village questionnaires. For collecting the information on contraceptive use National Family Health Survey-III asked the question form the eligible couples whether they are using any contraceptive method, if not then what is the reason for not using any contraceptive. Further the question was asked on intention of using any contraceptive method in future.

Cross-tabulation and multivariate analysis have been carried out to study the patterns of contraceptive use and their determinants. Individual level data for currently married women has been used for the analysis. Dependent variable is the current use of contraception which is dichotomous in nature- not using (0), and currently using (1). Analyses have been done by using Binary logistic regression analysis, which gives the positive and negative impact of each factor on dependent variable holding the other variables constant.

\section{RESULTS}

Table 1: Knowledge of family planning methods in Uttar Pradesh.

\begin{tabular}{|llll|}
\hline Method & Urban & Rural & Total \\
\hline Any method & 99.7 & 99.5 & 99.5 \\
\hline Any modern method & 99.7 & 99.5 & 99.5 \\
\hline Pill & 98.6 & 94.0 & 98.9 \\
\hline IUD & 96.3 & 85.4 & 95.1 \\
\hline Female sterilization & 99.5 & 98.7 & 98.9 \\
\hline Male sterilization & 96.3 & 89.6 & 91.2 \\
\hline Condom & 85.2 & 81.0 & 83.1 \\
\hline Any traditional method & 77.1 & 72.0 & 73.2 \\
\hline Rhythm/safe period & 73.7 & 67.5 & 69.0 \\
\hline Withdrawal & 48.1 & 40.6 & 42.4 \\
\hline Other method & 1.4 & 1.5 & 1.5 \\
\hline Number of women & 2160 & 6974 & 9134 \\
\hline
\end{tabular}

Source: National Family Health Survey -III, Uttar Pradesh, 2005-06

Lack of knowledge of contraceptive methods can be a most effective factor in using the different contraceptive methods. In NFHS-3, information was obtained on knowledge of different contraceptive methods by asking questions from eligible women. The family planning programme has succeeded in generating universal knowledge of family planning methods among the married couples. The table 1 shows that about $99.5 \%$ of 
the sample population of Uttar Pradesh knows about at least one contraceptive method. High awareness of family planning methods is also noticeable in rural areas with 99.5\%. Among the various contraceptive methods female sterilization is most widely known method (98.9\%) and is followed by oral pill which is known to $98.9 \%$ population of Uttar Pradesh. IUD is third most commonly known contraceptive, about $95.1 \%$ eligible couples were aware about this contraceptive method, $83.1 \%$ of the population had knowledge about condom. The rural urban variation in terms of knowledge of different contraceptive methods is evident from the table. Though the knowledge about contraception is universal in the rural areas as well but the knowledge about IUD, male sterilization, rhythm or safe period and withdrawal method are less familiar to them than their urban counterparts.

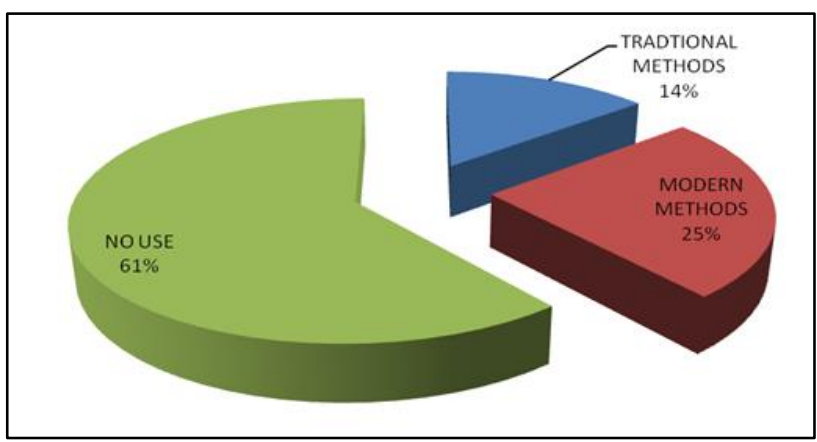

Figure 1: Prevalence of contraceptive methods in Uttar Pradesh, 2005-06.

\section{Pattern of contraceptive prevalence rate (CPR) in Uttar Pradesh}

The contraceptive prevalence rate varies according to the different socio-economic background characteristics of the women. The place of residence has immense impact on utilization of family planning methods especially among currently married women in India. The figure shows that current use of contraceptive methods among married women is higher in urban areas (56\%) as compared to rural areas $(39 \%)$. In urban areas modern contraceptives methods are more widespread (42\%) in comparison to rural areas $(25 \%)$.

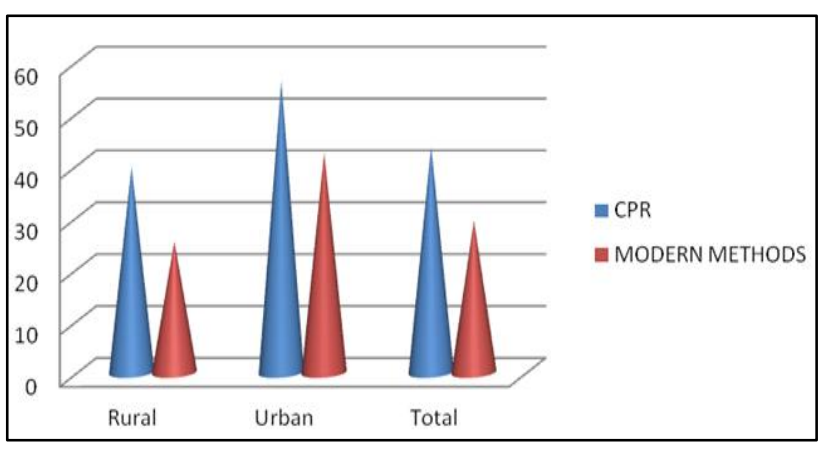

Figure 2: Contraceptive prevalence rate among currently married woman in Uttar Pradesh 2005-06.
It is evident from the table that in the early age women do not use contraception. In the age group 15-19 only $14 \%$ currently married women use any type of family planning method. As the age increases the use of contraceptive also increases, this might be because the older women are more close to complete desired family size. With increasing age, the women are more exposed to the knowledge about contraception. This rate increases up to $59 \%$ in the age bracket of 35-39 after that age bracket once again use of contraception declines. The use of modern contraceptive methods is also very low among all the age groups of women in Uttar Pradesh. It can be seen from the table that only $6 \%$ of women in the age group 15-19 use modern methods of family planning.

In Indian society the use of contraceptive method is rather complex. There are some religious groups who have less social access to these family planning methods. The table reveals that Sikh women have the highest adoption rate of contraception with $75 \%$, followed by Hindu women. Muslim women register lowest contraceptive prevalence with only $29 \%$, and only $20 \%$ of them are using modern methods.

Scheduled caste and scheduled tribes are the most vulnerable sections of the society. Women belong to Schedule Caste and Scheduled Tribes utilize only $42 \%$ and $38 \%$ of contraceptives respectively. The 'Gap' of knowledge and acceptance of family planning methods substantially decreases with increasing level of education of the women. Among the currently married women who have completed high school, the use of contraception is $58 \%$ whereas it is only $39 \%$ among illiterate women in Uttar Pradesh.

The standard of living index is an economic indicator to know about the acceptance of family planning methods. In Uttar Pradesh, contraceptive prevalence rate (CPR) increases from $34 \%$ for the women who have a low standard of living index (SLI) to $63 \%$ for women with high SLI. Though the utilization of modern contraceptive is higher among women with high standard of living, even then around $51 \%$ of currently married women are not using these modern methods.

The number of living children also affects the current use of any contraceptive method. It is clear from the table that contraceptive prevalence rate is only $8 \%$ among the women who do not have any child; this is mainly because every woman wants to have some children. Percentage use of contraception increases as the number of children increases, after having 4 children $53 \%$ women use contraceptive method. The use of modern contraceptive methods also increases with the increasing number of children but even then the use of these modern methods is very low in Uttar Pradesh. 
Table 2: Contraceptive prevalence rate $(\mathrm{CPR})$ in Uttar Pradesh, 2005-06.

\begin{tabular}{|c|c|c|}
\hline Predictors & $\operatorname{CPR}(\%)$ & $\begin{array}{l}\text { Any } \\
\text { Modern } \\
\text { Method \% }\end{array}$ \\
\hline \multicolumn{3}{|l|}{ Type of Residence } \\
\hline Rural & 39.7 & 25.3 \\
\hline Urban & 56.3 & 42.4 \\
\hline Total & 43.6 & 29.3 \\
\hline \multicolumn{3}{|l|}{ Age groups } \\
\hline $15-19$ & 14.5 & 6.3 \\
\hline $20-24$ & 26.7 & 15.5 \\
\hline $25-29$ & 44.2 & 28.9 \\
\hline $30-39$ & 59.1 & 41.1 \\
\hline $40-49$ & 46.3 & 33.6 \\
\hline \multicolumn{3}{|l|}{ Religion } \\
\hline Hindu & 46.3 & 30.9 \\
\hline Muslims & 29.6 & 20.5 \\
\hline Sikhs & 75.0 & 64.4 \\
\hline \multicolumn{3}{|l|}{ Ethnicity } \\
\hline Schedule Caste & 42.2 & 25.8 \\
\hline Schedule Tribe & 38.5 & 29.2 \\
\hline Other Backward Caste & 42.1 & 28.2 \\
\hline Others & 48.2 & 34.8 \\
\hline \multicolumn{3}{|l|}{ Level of Education } \\
\hline Illiterate & 39.7 & 25.8 \\
\hline $\begin{array}{l}\text { Literate but below middle } \\
\text { school }\end{array}$ & 40.1 & 28.2 \\
\hline Middle school complete & 46.5 & 30.6 \\
\hline $\begin{array}{l}\text { High school complete \& } \\
\text { above }\end{array}$ & 58.1 & 44.2 \\
\hline \multicolumn{3}{|l|}{ Standard of living } \\
\hline Low & 34.2 & 18.6 \\
\hline Medium & 39.9 & 26.1 \\
\hline High & 63.1 & 49.7 \\
\hline \multicolumn{3}{|l|}{ Number of living children } \\
\hline No Child & 8.4 & 4.3 \\
\hline 1 & 27.9 & 14.4 \\
\hline 2 & 49.1 & 35.3 \\
\hline 3 & 49.9 & 40.2 \\
\hline $4+$ & 53.6 & 35.5 \\
\hline
\end{tabular}

Source: National Family Health Survey -III, Uttar Pradesh: 2005-06

\section{Factors influencing current use of contraceptive methods}

Socio-economic differentials do not give a clear picture of factors affecting utilisation of contraceptive methods. In a bi-variate analysis the influence of each factor on contraceptive use is analysed without controlling the effect of other factors. The results from such a bivariate analysis could be misleading. Therefore, to know the true significance of each factor, it is necessary to control the effects of other factors through a multivariate analysis. As current use of contraception is measured in the binary form-either yes or no- thus data have been analysed by using logistic regression analysis. The figures presented in the table 3 are the 'odds ratios'. For a categorical explanatory variable, the odds ratio shows the probability of using contraception for a given category (e.g. rural) relative that for the reference category (e.g. urban).

Results from logistic regression explains that currently married women from rural area are $32 \%\left(\mathrm{OR}=0.681^{* *}\right)$ significantly less likely to use contraception than urban women, if other variable are constant. The main reason behind low prevalence of contraception in rural areas is the lack of availability and awareness about the family planning. It is evident from the table that women's age is positively related with current use of contraception. But there is non-linear relationship wherein the use of contraception increases with higher age groups at decreasing rate. If age group 30-39 of currently married women is a reference category (1) then women in the age group of 15-19 are only .04 times using contraception, likewise probability of using contraception increases with increasing age group up to 35-39, but for women in 45-49 age group probability of using contraception again decreases. It is because of many women cross their reproductive span at this age group, and there is no use of contraception.

There are certain social groups which have less access to contraceptives, have less education and awareness about the family planning methods. Among these social groups the contraceptive prevalence rate is found to be very low. In Uttar Pradesh, the likelihood of using contraception is $46 \%(\mathrm{OR}=0.549)$ lower among Muslims compared with Hindus. Sikhs are 1.34 times more likely to use contraception relative to reference category (Hindus). There are certain social norms in different religions, which affect the use of contraceptive methods. The odds of using contraception are $12 \%(\mathrm{OR}=0.883 *)$ lower among schedule tribe than the reference category (schedule caste, OR=1). Other Backward Caste and Others are $8 \%$ and $20 \%$ more likely to use contraception then schedule caste, holding the other determinants constant.

If we take illiterate women as reference category then regression analysis reveals that women below middle school are $30 \%(\mathrm{OR}=1.302 * * *)$ and women with middle complete are $67 \%\left(\mathrm{OR}=1.669^{* * *}\right)$ more likely to use the contraceptive methods than the reference category. High school completed women are $90 \%$ more likely to use contraception than the illiterate women in Uttar Pradesh. This shows a positive relationship between education of women and current use of contraceptive methods among married women. As per the analysis, if medium standard of living index is reference category then women belonging to low SLI are $44 \%\left(\mathrm{OR}=0.569^{*}\right)$ significantly less likely and high standard of women are $60 \%(\mathrm{OR}=1.599 *)$ significantly more likely to use contraception, if other factors remaining the same. 
Results from logistic regression analyses show that women with increasing parity use more contraception. Women at parity 3 are 19 times $\left(\mathrm{OR}=19.327^{*}\right)$ more likely to use contraception than the women with no children. But women at parity 4 or above are only 17 times higher than the reference category (women with no children). This percentage is lower because women with parity 4 and above also cross their reproductive span thus there is no need of using contraception anymore.

Table 3: Estimates of odds ratio of current use of contraception derived from logistic regression, Uttar Pradesh, NFHS-3.

\begin{tabular}{|c|c|c|c|}
\hline Predictors & B Coefficient & S.E. & Exp. (B), Odds Ratio \\
\hline \multicolumn{4}{|l|}{ Type of Residence } \\
\hline \multicolumn{2}{|l|}{ Urban (R) } & & 1 \\
\hline Rural & -0.385 & 0.035 & $0.681 * *$ \\
\hline \multicolumn{4}{|l|}{ Age } \\
\hline \multicolumn{2}{|l|}{$35-39(\mathrm{R})$} & & 1 \\
\hline $15-19$ & -3.227 & 0.009 & $0.04 *$ \\
\hline $20-24$ & -1.983 & 0.049 & $0.138^{*}$ \\
\hline $25-29$ & -0.885 & 0.042 & 0.413 \\
\hline $30-34$ & -0.207 & 0.042 & $0.813^{*}$ \\
\hline $40-44$ & -0.129 & 0.047 & 0.879 \\
\hline $45-49$ & -0.425 & 0.05 & $0.654 * *$ \\
\hline \multicolumn{4}{|l|}{ Religion } \\
\hline \multicolumn{2}{|l|}{ Hindus (R) } & & 1 \\
\hline Muslims & -0.599 & 0.04 & 0.549 \\
\hline Sikhs & -0.146 & 0.201 & 0.864 \\
\hline \multicolumn{4}{|l|}{ Ethnicity } \\
\hline \multicolumn{2}{|l|}{ Scheduled Caste (R) } & & 1 \\
\hline Scheduled Tribe & -0.125 & 0.056 & $0.883^{*}$ \\
\hline Other Backward Caste & 0.079 & 0.04 & $1.082 *$ \\
\hline Others & 0.184 & 0.041 & $1.202 *$ \\
\hline \multicolumn{4}{|l|}{ Education } \\
\hline \multicolumn{2}{|l|}{ Illiterate (R) } & & 1 \\
\hline Below Middle & 0.264 & 0.043 & $1.302 * * *$ \\
\hline Middle Complete & 0.512 & 0.063 & $1.669 * * *$ \\
\hline High School Complete & 0.643 & 0.057 & $1.903 * * *$ \\
\hline \multicolumn{4}{|l|}{ Standard of Living Index } \\
\hline \multicolumn{2}{|l|}{ Medium (R) } & & 1 \\
\hline Low & -0.564 & 0.032 & $0.569 *$ \\
\hline High & 0.469 & 0.038 & $1.599 *$ \\
\hline \multicolumn{4}{|c|}{ Number of Living Children } \\
\hline \multicolumn{2}{|c|}{$0(\mathrm{R})$} & & 1 \\
\hline 1 & 1.578 & 0.117 & $4.847 *$ \\
\hline 2 & 2.771 & 0.113 & 15.976 \\
\hline 3 & 3.412 & 0.114 & $19.327 *$ \\
\hline $4+$ & 3.158 & 0.115 & $17.52 *$ \\
\hline
\end{tabular}

* Sig. at less than $1 \%$ level of significance; ** Sig. at $5 \%$ level of significance; ***Sig. at $10 \%$ level of significance;

$(\mathrm{R})=$ reference category

\section{DISCUSSION}

India was the first country in the world to adopt population policies in 1951 and launching National Family Planning Programme in the following year.
Though fertility rates have come down but still the fertility rates are higher in comparison to the developed nations. $^{2}$ In India, knowledge of contraceptive is universal but there exists a wide gap between knowledge and acceptance. ${ }^{3}$ 
Empowered Action Group (EAG) states account for half of the country's population. Uttar Pradesh has been in the centre of various population stabilization programmes in India. In the last 65 years, Uttar Pradesh has experienced rapid population growth. ${ }^{6}$ In Uttar Pradesh the knowledge of contraception is almost universal, with $98 \%$ of currently married women having knowledge of at least one contraceptive method. ${ }^{7}$

Bi-variate analysis describe that contraceptive prevalence rate varies according to the different socio-economic background characteristics of the women. The place of residence has immense impact on utilization of family planning methods especially among women in India. Current use of contraceptive methods among married women is higher in urban areas (56\%) as compared to rural areas $(39 \%)$. Age of the women is an important demographic variable that affects women's contraceptive behavior. ${ }^{8,9}$ It is evident from the analysis that in the early age women do not use contraception. It might be because of lack of knowledge about the family planning methods and lower decision making power. Moreover, similar findings were cited in a study in Bangladesh that younger women are less likely to use family planning methods than older women. ${ }^{9}$

The Sikh women have the highest adoption rate of contraception with $75 \%$, followed by Hindu women. While, Muslim women register lowest contraceptive prevalence rate with only $29 \%$. Religion is said to be one of the important determinant influencing acceptance and use of contraceptive methods in India. ${ }^{10}$ Women belong to Schedule Caste and Scheduled Tribes are using less contraception than the women belong to others. Amongst the currently married women who have completed high school, the use of contraception is $58 \%$ whereas only $39 \%$ illiterate women use any contraceptive method in Uttar Pradesh. Female education is the most important factor in determining the female autonomy in reproductive decision. ${ }^{11}$ In Uttar Pradesh, contraceptive prevalence rate (CPR) increases from $34 \%$ for the poorest women to $63 \%$ for women with high standard of living index. The use of any contraceptive method is positively related to the standard of living index (SLI). ${ }^{12}$ Percentage use of contraception increases as the number of children increases, after having 4 children $53 \%$ women use contraceptive method. The most important factor in deciding the use of contraceptive methods is the son preference in India. ${ }^{10}$

Results from logistic regression reveal that currently married women from rural area are $32 \%$ significantly less likely to use contraception than urban women. Women's age is positively related with current use of contraception. In Uttar Pradesh, the likelihood of using contraception is $46 \%$ lower among Muslims compared with Hindus. Women below middle school are $30 \%$ and women with middle complete are $67 \%$ more likely to use the contraceptive methods than the illiterate women. The standard of living index of the household also shows a positive effect on use of contraception. ${ }^{12}$ Women belonging to low standard of living are $44 \%$ significantly less likely and high standard of women are $60 \%$ significantly more likely to use contraception, than women belong to medium SLI. The women with increasing parity use more contraception. Similar findings have been cited by some other studies. ${ }^{9}$

\section{CONCLUSION}

In Uttar Pradesh, contraceptive prevalence rate among currently married women is very low (43\%), and less than one third of currently married women using modern methods (only 29\%). There is a wide gap between knowledge and acceptance of contraceptives among currently married women in Uttar Pradesh. There are some socio-economic and demographic factors which affect the adoption of different family planning methods. Among all the determinants, women's education found to be the most important to affect the acceptance of contraceptives among currently married women.

In India National Family Planning Programme was launched in 1952, with the target to reduce fertility levels and stabilize population growth rates. Still after 63 years, we are far away from achieving our goals. Thus, there is an urgent need to strengthen the awareness among currently married women so that ultimate aim of reducing fertility rates can be achieved.

\section{Funding: No funding sources \\ Conflict of interest: None declared \\ Ethical approval: Not required}

\section{REFERENCES}

1. United Nations. Revised World Population Prospects Report. 2015.

2. Mohapatra A, Mishra CP, Gupta MK, Shivalli S, Mohapatra SC. A study on fertility pattern of recently delivered women in a rural area of Varanasi. Indian J Prev Soc Med. 2011;42:82-6.

3. Kulkarni MS. Women's exposure to mass media and use of family planning methods: A case study of Goa. Indian J Community Med. 2005;30:37-8.

4. International Institute for Population Sciences (IIPS). National Family Health Survey (NFHS-3), India, 2005-06.

5. Health Wise. Hindustan Times. Allahabad. 12 July 2009:14.

6. Singh JV. Family planning (IV chapter), Baseline facts- Uttar Pradesh, 1st edition. Government of Uttar Pradesh, Department of Medical Health and Family Welfare, Modern Printers. 2005;45.

7. International Institute for Population Sciences (IIPS). National Family Health Survey (NFHS-3), Uttar Pradesh, 2005-06.

8. Bhattacharya SK, Ram R, Goswami DN, Gupta UD, Bhattacharya K, Ray S. Study of unmet need for family planning among women of reproductive age 
group attending immunization clinic in a medical college of Kolkata. Indian $\mathbf{J}$ Community Med. 2006;31:73-5.

9. Barkat A, Rahman MU, Bose ML. Family planning choice behavior in urban slums of Bangladesh: An econometric approach. Asia Pac Popul J. 1997;12:17-32.

10. Chako E. Women's use of contraception in rural India: A village level study. Health and Place. 2001;41(3):529-45.

11. Saleem S, Bobak M. Women's autonomy, education and contraception use in Pakistan: a national study. Reproductive Health. 2005;2:8.
12. Patro BK, Kant S, Baridalyne N, Goswami AK. Contraceptive practice among married women in a resettlement colony of Delhi. Health and Population: Perspectives and Issues. 2005;28(1):916.

Cite this article as: Kerketta S, Kumar A. Knowledge of family planning and current use of contraceptive methods among currently married women in Uttar Pradesh, India. Int J Community Med Public Health 2015;2:449-55. 